\title{
Intramedullary Spinal Cord Metastasis From Rectal Cancer
}

\author{
Kyung Ho Yang, Hye Ran Lee, Seong Yoon Yi, Joo Hyuk Jung, Seung Hee Kang ${ }^{1}$, Pyong Hwa Choi ${ }^{2}$ \\ Departments of Internal Medicine, ${ }^{1}$ Radiation Oncology, and ${ }^{2}$ General Surgery, Inje University Ilsan Paik Hospital, Ilsan, Korea
}

Intramedullary spinal cord metastasis (ISCM) is an uncommon condition of the central nervous system (CNS) cause by systemic malignant tumors. Most ISCM cases are known to occur in patients with lung cancer and breast cancer; however, ISCM also very rarely occurs in patients with colorectal cancer. For the first time in Korea, we experienced a case of ISCM arising from rectal cancer, where a 75-year-old man presented with an abruptly-developed left-foot drop and numbness in both legs. The patient had lung metastases from rectal cancer that had been treated with chemotherapy. Magnetic resonance imaging revealed an intramedullary nodular lesion at the T12 level. ISCM was diagnosed and treated with steroids and radiotherapy. The patient's neurological symptoms were relieved for a while after treatment, but his condition deteriorated progressively. He died 4 months after ISCM had been diagnosed.

Keywords: Rectal neoplasms; Intramedullary spinal cord neoplasms; Radiotherapy

\section{INTRODUCTION}

Intramedullary spinal cord metastasis (ISCM) is a rare complication of cancer, affecting $0.1 \%-0.4 \%$ of all cancer patients $[1,2]$. It accounts for $2 \%-8.5 \%$ of central nervous system (CNS) metastases and $0.9 \%-5 \%$ of all spinal cord metastases. ISCM arises most commonly from small-cell lung cancer, but can also be seen in nonsmall-cell lung cancer, breast cancer, kidney cancer, melanoma, and lymphoma [2]. Until now, colorectal-cancer-related ISCM has been extremely rare, with only a few cases reported in the literature $[2,3]$. In Korea, colorectal-cancer-related ISCM has not been reported yet.

The most common symptom of ISCM is a rapidly progressive neurologic deficit. The overall prognosis is reported to be very poor; half of patients with ISCM die in the 3-4 months after the appearance of the first symptom [1-3]. However, in recent years, due to progress in diagnostic imaging, microsurgical techniques, and radiotherapy, the duration of survival and the quality of life

Received: July 8,2014 - Accepted: August 6, 2014

Correspondence to: Hye Ran Lee, M.D.

Department of Internal Medicine, Inje University Ilsan Paik Hospital, 170 Juhwa-ro, Ilsanseo-gu, Goyang 411-706, Korea

Tel: +82-31-910-9722, Fax: +82-31-910-7219

E-mail: leehr@paik.ac.kr

(C) 2014 The Korean Society of Coloproctology

This is an open-access article distributed under the terms of the Creative Commons Attribution NonCommercial License (http://creativecommons.org/licenses/by-nc/3.0) which permits unrestricted noncommercial use, distribution, and reproduction in any medium, provided the original work is properly cited. have improved because of early detection and prompt management, even in patients with ISCM $[1,2]$.

We report on a patient with an intramedullary metastasis from advanced rectal cancer coexisting with lung metastases and diagnosed by magnetic resonance imaging (MRI). Our case is the first report of ISCM arising from colorectal cancer in Korea.

\section{CASE REPORT}

A 75-year-old man came to the hospital, complaining of left-foot drop and numbness in both legs that had appeared 3 days before. He also had left lower leg and foot pain. He had no recent history of trauma in the legs. The symptoms progressed rapidly over 3 days. The patient had been diagnosed with rectal cancer 4 years earlier, which was found on the proximal $2.5 \mathrm{~cm}$ from the anal verge. At that time, colonoscopic biopsy confirmed a moderately-differentiated adenocarcinoma; the patient underwent neoadjuvant chemoradiation therapy, followed by Mile's surgery. During surgery, a pathological specimen showed no residual tumor on the anus and distal rectum; however, submucosal fibrosis, reactive epithelial changes, and acellular mucin pools were seen. No lymphnode metastases were found in 20 regional lymph nodes $(0 / 20)$. The patient had maintained a disease-free status after surgery, but 20 months after the operation, multiple metastatic nodules of the lung were found on computed tomography of the chest (Fig. 1). He was treated with palliative chemotherapy with FOLFOX regimen (5-FU, oxaliplatin, leucovorin). Even after first line chemotherapy, his disease did not improve. Thus, the chemotherapeutic 

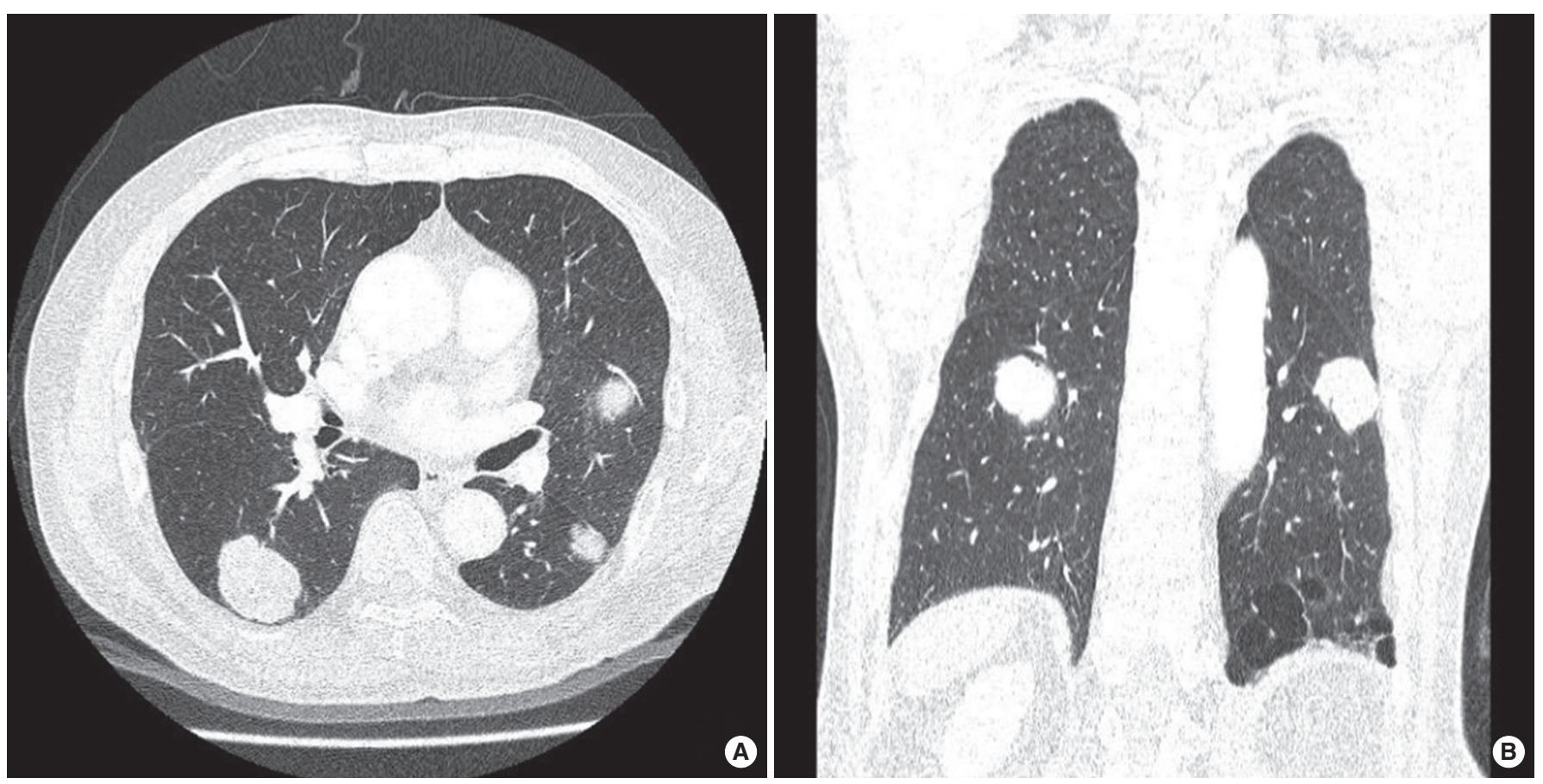

Fig. 1. Chest computed tomography (CT): an axial view (A) and a coronal view (B) on chest CT, showing multiple metastases on both lungs.

regimen was switched to the second line, capecitabine. After 4 months, multiple metastatic nodules grew again, and new nodules were found in the patient's lungs. We changed the chemotherapeutic regimen again to FOLFIRI (5-FU, irinotecan, leucovorin). The lung metastatic nodules responded to third line chemotherapy; however, they decreased rather than disappearing. His disease maintained a stable status over 11 months, after which it no longer responded to chemotherapy and continued to progress. While the patient was receiving supportive care, the symptoms of left-foot drop and numbness in both lower legs developed suddenly.

On physical examination, the patient had left dominant lowerextremity motor weakness (left leg motor, Gr2; right leg motor, Gr4) while his upper-extremity muscle power was intact. He felt numbness in the left leg and the right leg, extending to the left hip and the right knee. He exhibited an absence of deep tendon reflex on both knee joints and both ankle joints. He had pain in his left foot, along with the foot drop. A thoracolumbar MRI revealed a peripheral enhanced intramedullary nodular lesion $(0.9 \mathrm{~cm} \times 0.9$ $\mathrm{cm} \times 2.8 \mathrm{~cm}$ ) at the T12 level and diffuse spinal-cord signal change with a bulging contour at the thoracic spine level, suggesting diffuse intramedullary metastasis or secondary change due to a mass on the T12 spine (Fig. 2). A cerebrospinal fluid (CSF) analysis showed no abnormal findings, and CSF cytology was negative. The patient was immediately treated with steroids and radiation therapy (39 Gy in 13 fractions) to relieve his symptoms. His symptoms improved for awhile after this treatment; however, his condition deteriorated progressively, and he finally became unconscious. He died 4 months after the ISCM had been diagnosed.

\section{DISCUSSION}

ISCM arising from colorectal cancer is extremely rare, and only 12 cases have been reported so far in the literature in English [4-6]. Moreover, to the best of our knowledge, colorectal-cancer-related ISCM has not yet been reported in Korea. According to one Korean publication, ISCM was found in 12 cases from 1995 to 2006, but all 12 ISCMs arose from breast cancer or lung cancer [1].

Three pathological mechanisms have been suggested for ISCM $[2,3]$. Hematogenous spread is believed to be the most common spread route, as the common coexistence of pulmonary metastasis and brain metastasis in ISCM patients supports the possibility of spread through an arterial route $[2,3]$. Therefore, dissemination through arterial routes from the carcinoma with adjacent vessels has been speculated to be a major mechanism for hematogenous spread $[2,7]$. Another hematogenous spread route is considered to be the vertebral venous plexus, extending from the pelvis to the cranial venous sinuses $[1,4]$. This second mechanism is associated with meningeal seeding. Tumor cells from carcinomatous meningitis may infiltrate the vessels, penetrate the spinal cord, and eventually invade the spinal-cord parenchyma [2]. The third mechanism is related to dissemination though direct extension of a metastatic tumor from the spinal extradural space, CSF, and nerve roots [2].

The fact that this patient had multiple pulmonary metastatic nodules and negative CSF findings suggests that ISCM in this patient could have resulted from hematogenous dissemination. In a report by Ogino et al. [8], four of eight colorectal-related ISCM 

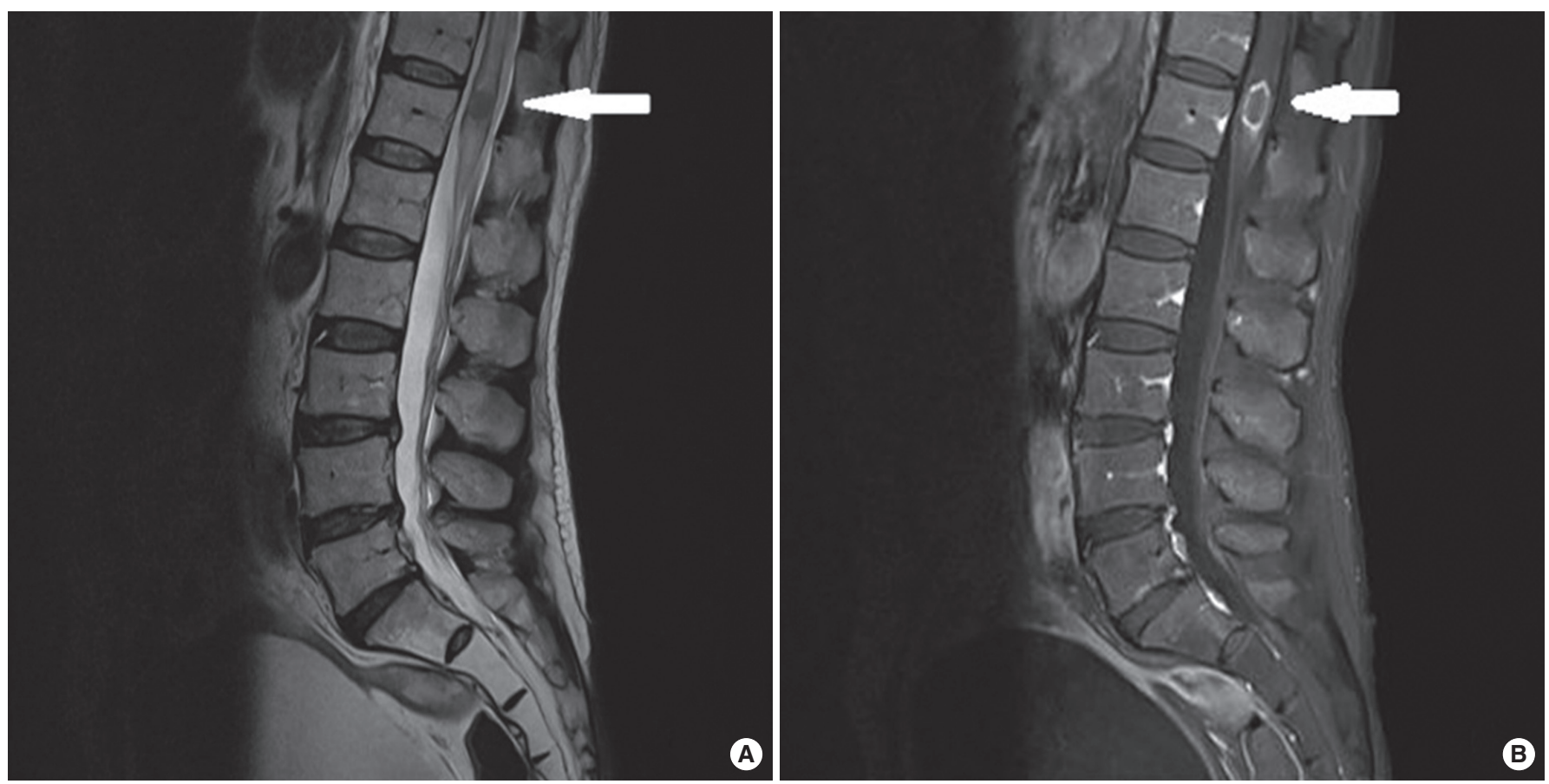

Fig. 2. Spine magnetic resonance imaging: (A) T2-weighted view showing solitary intramedullary nodular lesion at the T12 level and (B) contrast-enhanced T1-weighted image in the patient, indicating an intramedullary lesion with a peripheral enhancing pattern at the T12 level (The arrow indicates the ISCM lesion).

cases exhibited lung metastasis, indicating that colon cancer patients with lung metastasis had a higher risk of ISCM than those without it. Patients with ISCM are usually in an advanced state of the disease with other metastases. In particular, $25 \%-60 \%$ of patients with a brain metastasis showed ISCM at the time of diagnosis $[2,3,9]$. In contrast, in several studies, patients with other vertebral metastases were observed to have few brain metastases [2]. That our patient had a brain metastasis was not clear at the time ISCM was diagnosed because a work-up for brain metastasis had not been performed. However, he did not show any symptoms or signs of a brain metastasis when ISCM was diagnosed.

The clinical presentation of ISCM arises from symptoms of spinal-cord invasion. Because the clinical features of cord compression and cord invasion are very similar [2], distinguishing a intramedullary metastasis from an extradural spinal metastasis through clinical presentation alone is difficult. Weakness and numbness are the most common symptoms of ISCM $[1,2,10]$. Weakness is presented in $91 \%$ of ISCM patients while $82 \%$ of such patients exhibit numbness. The duration of symptoms before diagnosis ranges widely, from 1 day to 2 years (median, 52 days) [2]. This patient presented with left-foot drop and numbness in both lower legs that had lasted for 3 days.

Currently, ISCM is mainly diagnosed through an imaging study. MRI has high sensitivity when it comes to identifying spine lesions [2]. Therefore, MRI should be recommended for a cancer patient who presents with a sudden onset of motor weakness, sensory loss, or severe back pain. According to several studies, positron emission tomography (PET) is helpful in diagnosing ISCM. When MRI is contraindicated, PET may be considered as an option to evaluate the presence of this condition [11]. Histological confirmation may not be necessary because imaging studies have high sensitivity and many patients with ISCM exhibit poor performance status. We were able to diagnose this patient as having ISCM via MRI.

ISCM has a very poor prognosis. The mean survival after diagnosis is reported to be 3 months, and the median survival of these patients is limited to $3-7$ months $[2,3,5]$. Our patient survived for 4 months after the ISCM had been found. Unfortunately, a guideline for ISCM treatment has not been established because ISCM is a rare complication in cancer patients [2, 7]. Surgical resection can be considered in selected cases. The therapist must consider several factors, including general performance, medical condition, tumor size, number of tumors, and leptomeningeal metastasis. The survival time of patients treated with a surgical resection was reported to be nearly two times that of other patients [2]. Because those patients exhibited good performance and medical status, a surgical resection may be considered as an option for patients with a single ISCM and good performance status. In other cases, more conservative approaches, such as radiation therapy, steroids, and chemotherapy, can be applied for ISCM patients to improve their quality of life. In patients treated with conservative measures, $50 \%$ improve, $28 \%$ exhibit no change, and 
$22 \%$ deteriorate. In patients who undergo a surgical resection, improvement is seen in $77 \%$ of the cases and no change in 23\% [7]. Steroid therapy is not considered a single treatment and has no additional survival benefit $[2,5]$. However, in patients with rapidly-progressive symptoms of cord compression, it may decrease pain and neurological dysfunction [12]. Chemotherapy has been used in combination with radiotherapy or surgical resection in some chemosensitive tumors, such as small-cell carcinomas and hematological malignancies $[7,13]$. Radiation therapy has been the treatment of choice in many advanced ISCM cases, as this can suppress the tumor and slow down the progression of neurological symptoms. The best responses can be observed in radiosensitive tumors if treated very early in the course of presentation. However, such an approach would be ineffective in radio-resistant tumors [5]. In a recent study, after radiotherapy, 10 of 18 patients (56\%) exhibited improvement in neurological deficits or relief from pain, but none achieved complete remission [5]. In several reports, radiation therapy of $20 \mathrm{~Gy}$ in 5 fractions to $40 \mathrm{~Gy}$ in 20 fractions was used $[1,5,14]$. Our patient was also treated with radiation therapy with steroids. This seemed to be effective for awhile, but his symptoms deteriorated progressively, and he ultimately died. Nevertheless, because few patients can undergo a surgical resection, radiation therapy is considered to be an important treatment for ISCM.

In conclusion, this report suggests that when a patient with metastasis on the lung or brain complains of neurological symptoms, the rare condition of ISCM should be considered. MRI and/or PET can help to diagnose ISCM. Treatment may involve surgical resection, radiotherapy, or chemotherapy. Regardless of treatment, the prognosis for patients with ISCM is very poor.

\section{CONFLICT OF INTEREST}

No potential conflict of interest relevant to this article was reported.

\section{REFERENCES}

1. Lee SS, Kim MK, Sym SJ, Kim SW, Kim WK, Kim SB, et al. Intramedullary spinal cord metastases: a single-institution experience. J Neurooncol 2007;84:85-9.
2. Kalayci M, Cagavi F, Gul S, Yenidunya S, Acikgoz B. Intramedullary spinal cord metastases: diagnosis and treatment: an illustrated review. Acta Neurochir (Wien) 2004;146:1347-54.

3. Costigan DA, Winkelman MD. Intramedullary spinal cord metastasis: a clinicopathological study of 13 cases. J Neurosurg 1985; 62:227-33.

4. Grasso G, Meli F, Patti R, Giambartino F, Florena AM, Iacopino DG. Intramedullary spinal cord tumor presenting as the initial manifestation of metastatic colon cancer: case report and review of the literature. Spinal Cord 2007;45:793-6.

5. Hashii H, Mizumoto M, Kanemoto A, Harada H, Asakura H, Hashimoto T, et al. Radiotherapy for patients with symptomatic intramedullary spinal cord metastasis. J Radiat Res 2011;52:641-5.

6. Vassiliou V, Papamichael D, Polyviou P, Koukouma A, Andreopoulos D. Intramedullary spinal cord metastasis in a patient with colon cancer: a case report. J Gastrointest Cancer 2012;43:370-2.

7. Hrabalek L. Intramedullary spinal cord metastases: review of the literature. Biomed Pap Med Fac Univ Palacky Olomouc Czech Repub 2010;154:117-22.

8. Ogino M, Ueda R, Nakatsukasa M, Murase I. Successful removal of solitary intramedullary spinal cord metastasis from colon cancer. Clin Neurol Neurosurg 2002;104:152-6.

9. Dam-Hieu P, Seizeur R, Mineo JF, Metges JP, Meriot P, Simon H. Retrospective study of 19 patients with intramedullary spinal cord metastasis. Clin Neurol Neurosurg 2009;111:10-7.

10. Kaballo MA, Brennan DD, El Bassiouni M, Skehan SJ, Gupta RK. Intramedullary spinal cord metastasis from colonic carcinoma presenting as Brown-Séquard syndrome: a case report. J Med Case Rep 2011;5:342.

11. Mostardi PM, Diehn FE, Rykken JB, Eckel LJ, Schwartz KM, Kaufmann TJ, et al. Intramedullary spinal cord metastases: visibility on PET and correlation with MRI features. AJNR Am J Neuroradiol 2014;35:196-201.

12. Gasser TG, Pospiech J, Stolke D, Schwechheimer K. Spinal intramedullary metastases. Report of two cases and review of the literature. Neurosurg Rev 2001;24:88-92.

13. Fakih M, Schiff D, Erlich R, Logan TF. Intramedullary spinal cord metastasis (ISCM) in renal cell carcinoma: a series of six cases. Ann Oncol 2001;12:1173-7.

14. Park HK, Chang JC. Review of stereotactic radiosurgery for intramedullary spinal lesions. Korean J Spine 2013;10:1-6. 